Submitted to as an invited article to the Special Issue in Polymer "Probing Soft Matter by AFM" Guest Editors Andreas Fery, Georg Krausch, Georg Papastavrou

\title{
Recording Stretching Response of Single Polymer Chains Adsorbed on Solid Substrates
}

Lucie Grebikova $^{1}$, Milad Radiom ${ }^{1}$, Plinio Maroni ${ }^{1}$, A. Dieter Schlüter ${ }^{2}$, Michal Borkovec ${ }^{1, *}$

${ }^{1}$ Department of Inorganic and Analytical Chemistry, University of Geneva, Sciences II, 30 Quai Ernest-Ansermet, 1205 Geneva, Switzerland

${ }^{2}$ Department of Materials, Institute of Polymers, Swiss Federal Institute of Technology, ETH Zurich, HCI J 541, 8093 Zurich, Switzerland

*Corresponding author: Email: michal.borkovec@unige.ch 


\begin{abstract}
Various techniques to measure the force-extension relationship of individual polymer chains with the atomic force microscope (AFM) are compared. Reliable stretching force profiles can be obtained with the nano-handling technique, which involves imaging of the measured individual polymer molecules prior and after the force experiment. Results originating from the classical pulling technique, which relies on dense adsorbed polymer layers, must be interpreted with care. Comparison with the nanohandling technique reveals that the stretching response of individual molecules can be obtained with the pulling technique, provided the adsorbed polymer layer is dilute. For denser adsorbed layers, such experiments may not reflect the correct stretching response. The discrepancies seem to be related to polymer-polymer interactions and entanglements within the adsorbed layer. AFM imaging of the adsorbed layers can provide an indication concerning the relevance of such entanglement effects. Similar caveats apply to fishing experiments, where polymers are adsorbed to the AFM tip and not to the substrate.
\end{abstract}

\title{
Graphical Abstract
}

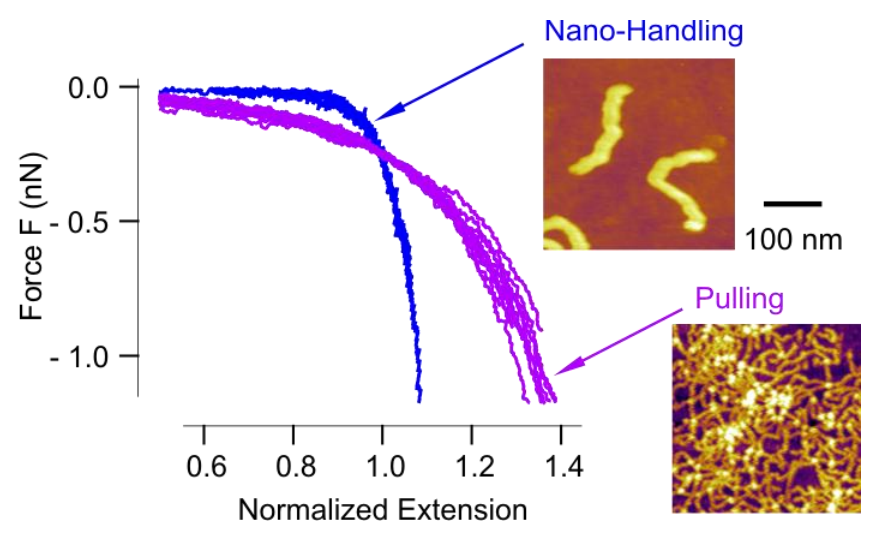

Keywords: Dendronized Polymers, Force Spectroscopy, Atomic Force Microscopy, Single Molecule, Polymer Elasticity 


\section{Introduction}

The atomic force microscope (AFM) has revolutionized our capabilities to image, probe, and manipulate nanoscopic objects on surfaces, especially for soft and polymeric materials. Among the different applications of this technique, which include single molecule imaging [1-3], measurements of forces and friction [4-7], the possibility to probe and manipulate single macromolecules seems most exciting [8-16].

Among these developments, the so-called single molecule force spectroscopy became a widely used tool to investigate the mechanical properties of single macromolecules, their interaction with surfaces, and chemical reaction kinetics on a single molecule level [10-26]. The technique relies on a configuration where the substrate and the tip are bridged with a single polymer molecule, and subsequent analysis of the measured force-distance curves, normally upon retraction, but eventually also upon approach. Two main classes of events can be normally observed, namely pulling and peeling. A pulling event resembles a spike in the force curve, and ideally corresponds to the forceextension relationship of a single polymer molecule, which is firmly attached to the substrate and to the tip [5,12,20,27-29]. Such force profiles can be interpreted by the freely jointed and worm-like chain models including chain elasticity. Recently, such experiments were also carried out with two AFM-tips, which further provide time-dependent properties of a single polymer chain [23]. A peeling event is characterized with a plateau in the force curve, which reflects the force that is necessary to peel off the adsorbed molecule [30-36]. These forces reflect the affinity of the molecules to the surface, and can be related to the type of substrate and its charge. Peeling and pulling events also may occur in succession, and their relative occurrence can be sometimes tuned through the surface properties or its coverage [30,37]. A series of spikes resembling teeth of a saw is normally interpreted as the unwrapping of individual macromolecule domains, which are frequently observed for muscle proteins (e.g., tintin, tenascin) [11,38]. A series of peeling events, which resemble a staircase, are typically due to the peeling of several molecules from the surface, even though they could also be caused by a combination of sliding and peeling [31,32,37,39,40]. Frequently, however, several of these events occur in combination, and the respective force profiles have complex, random appearances, which are difficult to interpret. The focus of this article is to show how single-molecule stretching response can be obtained from pulling events, while peeling events will not be discussed further.

To generate a single bridging polymer molecule between the substrate and the tip, various techniques have been proposed, see Fig. 1a. The nano-handling technique identifies a single adsorbed polymer molecule on the surface by AFM-imaging, and this molecule is probed with the AFM tip through repeated approach-retraction cycles [12,30]. A bridging event is identified by its force response. The conformation of the adsorbed molecule can be again probed by imaging after the force 
experiment. This approach is similar to a technique proposed earlier [41,42].The pulling technique is by far the simplest to realize and therefore the most popular $[5,20]$. This method relies on a substrate with an adsorbed polymeric layer, and this substrate is probed with repeated approach-retraction cycles. A bridging event is identified by spikes or plateaus in the force curve. The fishing technique is similar, but the polymers are adsorbed to the AFM tip $[35,36,43,44]$.

For all these techniques, whether the polymers will be picked up in a given conformation, and whether they will be stretched or peeled, depends strongly on the solution conditions, the nature of the substrate and the tip, and their surface functionalization. When the affinity of the polymer to the substrate is strong, no events will occur, while when this affinity is weak, peeling events will be observed. Pulling events normally predominate when the affinity is intermediate.

Once a molecule has been picked up, the retraction part of the force curve reveals whether it is being peeled or pulled. The mechanical response of individual polymer molecules can be only extracted from pulling events. Such a response can be obtained in different ways, see Fig. 1b. The lifting mode relies on approach-retraction cycles between large separation distances and the contact region $[5,20]$. In this mode, each bridging polymer detaches from the tip after each retraction. The contact clamping mode permits to access the reversibility of the stretching force response of an individual polymer $[17,27,29,45]$. Thereby, one retracts the tip from the surface only to the point that the polymer is being stretched, but without detaching it. The cycle is completed by approaching the tip again into contact. A hysteresis between the approach and retraction force curves indicates slow conformational transitions or refolding domains [46-48]. In the contact clamping mode, however, one cannot ascertain whether the same or a different polymer molecule is being extended within subsequent cycles. The non-contact clamping mode avoids this problem, since the tip is not approached to contact, whereby the polymer chain cannot be easily exchanged. Techniques involving combination of lateral and normal displacement of the tip were equally proposed, but they will not be pursued here [31,39].

Whether an observed pulling event originates from the stretching of a single or several polymer molecules is not necessarily obvious. Several tests were proposed that can be used to identify such events $[12,35,41,49]$. (1) When one uses the lifting mode, several pulling events may occur in succession upon retraction of the tip. Obviously, the last event likely corresponds to a single molecule. (2) Models of force-extension relationships suggest that the stretching force is only a function of the extension relative to the contour length. Therefore, the force profiles corresponding to single molecules will collapse upon a master curve, when plotted versus the normalized extension that corresponds to a given force, see Fig. 1c. (3) Performing fishing instead of pulling experiments will increase the probability to pick up a single molecule since the polymers are adsorbed on the tip only. (4) Most straightforward is to identify a single adsorbed polymer by imaging, and obviously, force 
extension experiments carried out in this configuration necessary involve single and isolated molecules only.

The aim of the present article is to demonstrate that not all these tests to identify the stretching response of a single molecule are trustworthy. Only (4) single molecule nano-handling seems reliable, but it may prove difficult to realize. On the other hand, the different tests including (1) last event, (2) master curve, and (3) fishing experiments, may prove unreliable and should be used with caution. We illustrate these facts with pulling experiments carried out with different polymers, and propose alternative tests to identify single molecule stretching response. The polymers used here include amino-functionalized dendronized polymers (DPs) of generation 1 and 3, which are abbreviated as PG1 and PG3, and poly(2-vinylpyridine) (P2VP). Their structures are summarized in Fig. 2.

\section{Experimental}

Polymers. Polymethacrylate-based DPs of different generations 1 and 3, denoted as PG1 and PG3, terminated with amine groups were synthesized with the attach-to route as described previously [50,51]. The polydispersity index of the side dendrons is about 1.01 with respect to the expected number of the amine groups for each repeat unit. The characterization of these DPs by gel permeation chromatography and AFM is discussed elsewhere [30,50,51]. DPs were dissolved in electrolyte solutions adjusted to $\mathrm{pH} 4.0$ with $\mathrm{HCl}$. The ionic strength was adjusted with $\mathrm{KCl}$, if necessary. Under these conditions, the amine groups are positively charged. Poly(2-vinylpyridine) (P2VP) was purchased from Sigma-Aldrich (Switzerland) with an average molecule mass of about $150 \mathrm{~kg} / \mathrm{mol}$. The polymer was dissolved in water adjusted to $\mathrm{pH} 3.0$ with $\mathrm{HCl}$ at a concentration of $100 \mathrm{mg} / \mathrm{L}$. The solution was diluted whenever necessary, whereby pH 3.0 was maintained. At these conditions, the polymer is also fully charged [1].

Substrates. Experiments were carried out with bare mica, bare silica, functionalized mica, and functionalized silica. Mica surfaces were obtained by cleaving high-grade mica (Plano Wetzlar, Germany) with adhesive tape. Amino-functionalized (AF) mica was obtained by placing the freshly cleaved mica sheet in an evacuated glass container aside a $30 \mu \mathrm{L}$ drop of 3(ethoxydimethylsilyl)propylamine (Sigma-Aldrich, Switzerland) for $12 \mathrm{~h}$. Epoxy-functionalized (EF) silica was prepared with naturally oxidized silicon wafers from Silchem (Germany). The wafers were cleaned for 20 min with piranha solution, which consists of a mixture of $\mathrm{H}_{2} \mathrm{SO}_{4} 98 \%$ and $\mathrm{H}_{2} \mathrm{O}_{2} 30 \%$ in a volumetric ratio of 3:1. The wafers were washed with Milli-Q water, dried in a stream of nitrogen, and placed in an evacuated container aside a $150 \mu \mathrm{L}$ drop of (3-glycidoxypropyl)dimethylethoxysilane (Sigma Aldrich) for $2 \mathrm{~h}$. The contact angles of the different substrates were extracted from 
digital photographs of sessile drops of pure water. They were $51^{\circ}$ for EF-silica and $68^{\circ}$ for AF-mica. For bare silica, the contact angle was $38^{\circ}$, while bare mica was fully wetted. All experiments were carried out at room temperature of $23 \pm 2^{\circ} \mathrm{C}$.

High-resolution AFM imaging. Imaging in air was performed with a Cypher (Asylum Research, Santa Barbara, CA). This instrument was also used to carry out all subsequent AFM experiments. High-resolution images were acquired in air in amplitude modulation mode with super sharp silicon tips (SSS-NCHR, Nanosensors, Neuchatel, Switzerland). These cantilevers have a nominal tip radius below $5 \mathrm{~nm}$, a spring constant of $42 \mathrm{~N} / \mathrm{m}$ and a resonance frequency in air around $330 \mathrm{~Hz}$. Images were obtained near the resonance frequency of the cantilevers with a scanning speed of $300 \mathrm{~nm} / \mathrm{s}$ with a free oscillation amplitude (FOA) of about $15 \mathrm{~nm}$. Images were also acquired in liquid with highresolution scanning probe microscopy probes with a spike on the silicon tip (Hi'Res-C19, MikroMasch, Wetzlar, Germany). The spike has a radius of about $1 \mathrm{~nm}$, and the cantilever has a spring constant of $0.5 \mathrm{~N} / \mathrm{m}$ and a resonance frequency around $65 \mathrm{kHz}$ in air. Images were acquired near the resonance frequency of the cantilevers with a scanning speed of $250 \mathrm{~nm} / \mathrm{s}$. The FOA was adjusted to around 5-10 $\mathrm{nm}$. Images of P2VP were acquired with Biolever mini cantilevers with silicon tips (BL-AC40TS, Olympus, Japan). These tips have a nominal tip radius $<10 \mathrm{~nm}$ and resonance frequencies around $30 \mathrm{kHz}$ in water and around $130 \mathrm{kHz}$ in air. The spring constants were in the range of $0.07-0.15 \mathrm{~N} / \mathrm{m}$ as determined in air by the thermal fluctuation method. The images were acquired in liquid close to the resonance frequency of the cantilevers with a scanning speed of $180 \mathrm{~nm} / \mathrm{s}$. The FOA was set to about $10 \mathrm{~nm}$.

Nano-handling. Biolever mini cantilevers described above were used. They were cleaned in a UVozone cleaner (PSD Pro, Novascan, Ames, USA) for $20 \mathrm{~min}$ in an oxygen-enriched atmosphere and epoxy-silanized overnight with (3-glycidoxypropyl)-dimethylethoxysilane as described above. For the experiments with DPs, the substrates used were AF-mica or EF-silica. The substrates were incubated in polymer solutions for $40 \mathrm{~s}$ at concentrations of $4-6 \mathrm{mg} / \mathrm{L}$. This procedure ensured a sufficient coverage of DPs on the surface, while the overlap of different chains was minimized. Subsequently the sample was rinsed with water and dried with a stream of nitrogen. The substrates were again incubated in the respective electrolyte solution, and images were acquired in solution. Selected experiments were used to verify that the drying has no effect on the experimental results. The drying step was introduced as it simplifies the mounting of the cell substantially. The substrates were first imaged in liquid, whereby the scan rate was set to $4.9 \mathrm{~Hz}$, FOA about $20 \mathrm{~nm}$, and a set point corresponding to around $70 \%$ of the FOA. After stabilization of the image, the piezo excitation of the cantilever was stopped, and the tip was positioned over the end of a selected polymer. The contact clamping mode was used for these experiments, whereby vertical approach-retraction cycles through the contact region were then recorded with a sampling rate of $2 \mathrm{kHz}$, approach-retraction velocity of 
$200 \mathrm{~nm} / \mathrm{s}$, and a maximum deflection of $30 \mathrm{~nm}$. The initial pull-off distance was chosen of about one quarter of the molecular contour length, which was deduced from the image. Once a molecule is picked up, the pull-off distance is initially increased to obtain larger forces and extensions, which are then kept constant to record a sensible number of force curves. This process is continued until the molecule falls off, which normally happens after $2-10$ cycles. This protocol corresponds to the contact clamping mode (Fig. 1b). The piezo displacement and cantilever deflection were converted to force-distance curves, resulting in a noise in the force curves of about $0.01 \mathrm{nN}$. After the force measurements, the same region was imaged again in order to observe the displacement of the targeted molecule. Further technical details and interpretation of such nano-handling experiments are given elsewhere [12].

Experiments with P2VP were performed in a similar way. The cantilevers were silanized with dimethoxy(methyl)-octylsilane or (3-glycidoxypropyl)-dimethylethoxysilane. The polymer was adsorbed to bare mica or bare silica from a solution of a polymer concentration of $0.5 \mathrm{mg} / \mathrm{L}$ during 40 $\mathrm{s}$ in $1 \mathrm{mM} \mathrm{HCl}$ of $\mathrm{pH} 3.0$. The pulling experiments were carried out in the same solution without polymer, and the force profiles were recorded in the lifting mode.

Pulling and fishing experiments. For pulling experiments, the substrates were coated with polymers. The respective substrates were incubated in the appropriate polymer solution with a concentration of $80 \mathrm{mg} / \mathrm{L}$ for $40 \mathrm{~min}$, rinsed with pure electrolyte solution, then with pure water, and dried. The biolever mini cantilevers were epoxy-functionalized as described above. For the fishing experiments, the functionalized cantilevers were incubated in the respective polymer solutions in the same way. The excess solution was removed with an absorbing tissue and dried. The AFM cell was assembled with the respective substrate, filled with the appropriate electrolyte solution, and the pulling experiments were carried out with repeated approach-retraction cycles with a sampling rate of $2 \mathrm{kHz}$. The other parameters were the same as given above. The experiments were carried out in different modes. The lifting mode was used with pull-off distances of more than $200 \mathrm{~nm}$. Whenever possible, however, the molecules were clamped. In this case, the contact clamping mode was used with pull-off distances that were initially chosen to be $20-40 \mathrm{~nm}$. Once a molecule was picked up, the distance was further increased until larger forces and extensions were obtained. At the largest distance, the forces curves were recorded during several cycles, until the molecule would fall off. At that point, the pulloff distance was decreased again until another molecule was picked up. The non-contact clamping mode was carried out in the same way, except once the molecule was pulled to the maximum distance, the cantilever was only partially re-approached to the surface, typically $20-50 \%$ of the maximum distance, but not closer to the surface than about $20 \mathrm{~nm}$. Within these conditions, several cycles were recorded. The drift within the distance in these experiments was below $0.07 \mathrm{~nm} / \mathrm{s}$. 
Pulling experiments were also carried out with P2VP. The substrates were incubated in the polymer solution of a concentration of $100 \mathrm{mg} / \mathrm{L}$ during one hour. Afterwards, the substrates were rinsed in $1.0 \mathrm{mM} \mathrm{HCl}$ of $\mathrm{pH} \mathrm{3.0,} \mathrm{and} \mathrm{the} \mathrm{substrate} \mathrm{was} \mathrm{transferred} \mathrm{in} \mathrm{the} \mathrm{wet} \mathrm{state} \mathrm{to} \mathrm{the} \mathrm{AFM} \mathrm{fluid}$ cell. The experiments were carried out in a similar fashion as described above, except that octylfunctionalized cantilevers were also used. The latter functionalization was carried out with dimethoxy(methyl)-octylsilane.

\section{Results and Discussion}

This study compares the stretching force response of individual adsorbed polymer molecules with the same polymers picked up from adsorbed layers. First, we discuss results obtained with the nanohandling technique, as it allows studying the response of a single polymer. Subsequently, these results are compared with the pulling technique, where the polymer is being picked up from denser polymer layer adsorbed on a flat substrate. Finally, we use the fishing technique, where the molecules are adsorbed to the AFM tip. These techniques are visualized in Fig. 1a. These experiments were first carried out in the lifting mode, whereby the tip is pulled completely away from the substrate during each cycle. Alternatively, experiments were done by clamping the polymer molecules, whereby multiple force extension curves were recorded with the same molecule, either contacting the substrate in every cycle or not. In the latter two cases, we refer to contact or non-contact clamping. These different modes are summarized in Fig. $1 b$.

Most experiments were carried out with PG3, but further experiments were equally performed with PG1 and P2VP. Two ionic strengths were normally compared, namely $0.1 \mathrm{mM}$ and $100 \mathrm{mM}$. An epoxy-functionalized AFM tip was used for all force experiments with DPs, while for P2VP octylfunctionalization and epoxy-functionalization were used.

Nano-handling with PG3. The force response of single polymer molecules can be best studied with the nano-handling technique. Consider the situation where PG3 was adsorbed from a dilute aqueous solution of a polymer concentration around $5 \mathrm{mg} / \mathrm{L}$ of low ionic strength of $0.1 \mathrm{mM}$ to an EF-silica substrate during 40 seconds. An AFM image of the adsorbed molecules is shown in Fig. 3a. The tip is then positioned over a selected end of a polymer molecule (arrow) and a series of vertical approach and retraction force profiles is recorded, see Fig. 3c. The tip is only retracted to about $30 \mathrm{~nm}$. During several initial approaches, such as shown in (1), the polymer is not being properly picked up, as can be seen from the featureless retraction curve at larger distances. A later approach cycle (2) was successful, as the retraction curve shows the polymer being lifted from the surface. The following approach profile (3) shows the elastic response of the polymer. The polymer is now lifted up again, 
but as shown in the retraction curve (3), it is pulled further out. The elastic response during approach is shown in (4). By repeating these experiments, as in traces (5) and (6), the polymer is lifted up further away from the surface, and a longer stretching response can be recorded. Aside some covalent attachment points, the polymer backbone interacts with the substrate weakly, and thus the retractions also yield clean force extension curves. At one point, the polymer detaches (7). After detachment, the same region is imaged again, see Fig. 3b. One observes that the polymer is again adsorbed at virtually the same location, possibly due to the existence of preferential adsorption sites. In this way, force curves can be recorded with the same polymer upon approach and retraction. For some approach curves (4-6) the adsorbed polymer obstructs the contact to the substrate, which is only achieved at higher forces. When one uses the first cycles to lift the polymer further away from the surface, one often obtains force extension curves that cover a wider force range. On the other hand, when one pulls too far, one risks losing the polymer that was picked up. The contact clamping mode (Fig. 1b) works well, and by selecting several polymers on the surface, a large number of force profiles extending over a wide force range can be collected. Note that only about one fifth of the contour length of polymer is lifted in the nano-handling experiment, which suggests infrequent but strong attachment points, which probably are due to covalent bonds between the chain and the substrate.

The force $F(x)$ that is measured as a function of the distance $x$ is normalized by determining the distance $x_{i}$ that satisfies $F\left(x_{i}\right)=F_{0}$ where $F_{0}$ is an appropriately chosen threshold force (see Fig. 1c). The forces are then plotted versus the normalized extension $x / x_{i}$ in Fig. 3d. The threshold force is chosen to be $F_{0}=0.25 \mathrm{nN}$. The overlay of the force profiles in this representation suggests that the force response originates from single molecules $[12,49]$. One observes that the force profiles recorded upon approach and retraction collapse upon a common master curve, confirming that the extension process is fully reversible. This reversibility originates from the nearly entirely elastic response of the stretched polymer, whereby energy dissipation rate within the chain is small \{Radiom, $2013 \# 7384\}$. During such extension experiment, one might be probing polymer segments of different contour length, since upon approach the polymer may attach on the substrate differently. However, when these force profiles are plotted versus the normalized extension, these profiles collapse in spite of the different contour lengths (Fig. 1c).

The measured force curves were also fitted with the freely jointed chain (FJC) model including chain elasticity up to forces of $0.5-1.5 \mathrm{nN}$, depending on the situation. The extension of the molecule $x$ can be expressed as a function of the force $F$ by the relation $[12,49]$

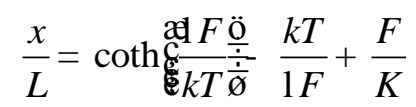


where $L$ is the contour length, $k$ the Boltzmann constant, $T$ the absolute temperature, 1 the effective Kuhn length, and $K$ the elasticity constant. This expression again illustrates that the force only depends on the relative extension $x / L$ and therefore lead to a master curve when plotted versus a normalized extension. The best fit of the force profile shown in Fig. 3d yields $1=0.24 \pm 0.04 \mathrm{~nm}$ and $K=17 \pm 4 \mathrm{nN}$. The values are in good agreement with the ones reported previously [12].

A similar experiment carried out at a high ionic strength of $100 \mathrm{mM}$ is shown in Fig. 4. Two different substrates were used, namely AF-mica and EF-silica. The molecules adhere more strongly to AF-mica than in the previous example, which leads to a retraction part that is very noisy. From these traces, no force extension profiles can be obtained. On the other hand, approach curves provide appropriate force profiles. The respective force extension profiles were again normalized, and they are shown in Fig. 4d. Comparison of the image before and after the experiment shown in Fig. 4a,b suggests that the molecule has been slightly displaced during the force experiment. Seemingly, there are some preferential adsorption sites, which cause the molecule to reabsorb in a similar, but different conformation. In the example illustrated in Fig. 3, change in the polymer conformation before and after the pulling experiment could even not be seen in the AFM image.

The same experiments were repeated with the EF-substrate. The approach and retraction curves resemble very much those shown in Fig. 3. The polymer interacts with the substrate more weakly, but attaches through covalent bonds strongly on few spots. The force extension curves are compared for the two different substrates in Fig. 4d. One observes that the results are identical within experimental error. This observation is a strong indication that the recorded response reflects the stretching of the individual molecule and is not influenced by any interactions with the substrate. The best fit of the force profile with the FJC model is shown in Fig. 4 and yields $1=0.27 \pm 0.06 \mathrm{~nm}$ and $K$ $=19 \pm 6 \mathrm{nN}$, which are again in good agreement with the values reported earlier [12]. This increase of the effective Kuhn length suggests a somewhat stiffer response of the chain at higher salt levels. This trend is reverse to the one expected from electrostatic interactions, which leads to decrease of the persistence length with increasing salt concentration [20]. We suspect that the observed trend is related to an increased hydrophobicity of the polymer backbone at higher salt levels [12]. Similar experiments employing other substrates have shown that the force profiles are reversible at an ionic strength of $100 \mathrm{mM}$ [12]. The minor deviations between the different force profiles are probably caused by the fact that the AFM-tip is not always vertically positioned above the attachment point of the polymer on the substrate, but that it is being pulled under different angles [39]. This effect should be more important at smaller extensions, but the force resolution is insufficient in this regime. This response was equally found to be independent of the pulling velocity in the range 50-200 nm/s.

While the nano-handling technique is eminently useful to obtain the stretching response of individual molecules, the technique has also its problems. The most important one surely is the proper 
choice and functionalization of the substrate. When the substrate is too strongly adhesive, the chain cannot be picked up. When adhesion is too weak, the chains peel from the substrate. For this reason, it was essential to use EF-silica for the experiments at low ionic strength, while EF-silica as well as AFmica could be used at high ionic strength. Further discussion concerning the proper selection of appropriate substrate and further details concerning the nano-handling technique are given elsewhere [12].

Pulling experiments with PG3. Let us now compare the results obtained by nano-handling with pulling experiments. The simplest way to perform such experiments is the lifting mode, see Fig. 1b. The tip is repeatedly brought into contact with the surface coated with PG3, and retracted completely to large distances. PG3 was adsorbed on these substrates from a more concentrated polymer solution of a concentration of $80 \mathrm{mg} / \mathrm{L}$ during $40 \mathrm{~min}$ and investigated at an ionic strength of $100 \mathrm{mM}$. Typical traces recorded on different substrates are shown in Fig. 5. The probability to detect a pulling event is about $1 \%$. While retraction events from bare mica and AF-mica can be readily observed, they display a complex structure, and it is not possible to extract individual pulling events. However, EF-silica shows well pronounced pulling events. They are collected in Fig. 5d and compared with the force response measured by nano-handling. One observes that all traces measured by the pulling technique in the lifting mode are softer. However, these traces do not superpose, and therefore it remains unclear whether they correspond to single molecule events or not. Similar experiments were also carried out at an ionic strength of $0.1 \mathrm{mM}$, but no sensible force profiles could be extracted.

Well reproducible force profiles can be obtained in the contact clamping mode, see Fig. 1b. While PG3 adsorbs on bare mica too strongly, AF-mica is well suited. Typical series of force curves is shown in Fig. 6a (series 1). In this case, approach and retraction force profiles could be extracted, and they confirmed full reversibility of these stretching experiments. The resulting normalized force profiles are shown in Fig. 6b. These profiles are also in very good agreement with the results obtained by the nano-handling technique. For better readability of the figures, we use the respective FJC fit of the nano-handling results for comparison. Such pulling experiments can also be reproduced with different molecules, as illustrated with series 2 (Figs. 6c and 6d). In this case, one could only obtain retraction profiles, but they coincide within experimental error with series 1 . Since the same response is obtained within different series, this fact indicates that the response of single molecules is being recorded. Another possibility would be that within a given series one, two, or several molecules would be picked up. In this case, however, the traces would not coincide [52]. Similar experiments were also carried out at an ionic strength of $0.1 \mathrm{mM}$ (Fig. 6e). In this case, the force profiles could be only obtained from the approach traces, but they also coincide with the results obtained by the nanohandling technique. This finding confirms that for the present substrate, one can obtain reliable single molecule force profiles from the pulling technique. However, an interesting aspect is that the 
clamping experiments typically reach larger forces than the lifting experiments. This difference could be due the fact that the clamping experiments will systematically select those molecules that have stronger attachment to the substrate and the tip.

The contact clamping mode cannot fully ascertain that successive cycles correspond to the same molecule. When the tip is in contact with the substrate, the adsorbed molecule could desorb, and another polymer adsorb. The non-contact clamping mode can be used to confirm that this process is unlikely, see Fig. 1b. When a molecule has been picked up, the cantilever is no longer brought to contact, but its motion is reversed at a separation of about $20 \mathrm{~nm}$. The respective results in a solution of an ionic strength of $100 \mathrm{mM}$ are summarized in Fig. 7. The sequence shown was recorded with the same polymer chain. The retraction curves also yield the force extension profiles. The comparison between the profiles measured during approach and retraction shown in Fig. $7 \mathrm{~b}$ further confirms that the process is fully reversible. Moreover, results obtained by pulling and nano-handling techniques again agree very well.

Similar non-contact clamping experiments were also carried out with PG3 adsorbed on EFsilica. By recording the approach and retraction traces shown in Fig. 8a, the normalized force profiles could be again extracted. Figure 8a compares such force profiles with the best FJC fit of the nanohandling results. In this case, however, one observes a substantial disagreement, whereby the forces profiles obtained by the pulling technique are substantially softer. Moreover, the approach and retraction profiles do not coincide, indicating lack of reversibility. Therefore, these force profiles measured on EF-silica do not reflect the stretching response of a single polymer, which is firmly attached on both sides. The traces obtained in the clamping mode now differ substantially from the nano-handling results. Moreover, they again lack reversibility. The supplement also illustrates that similar discrepancies are present in contact clamping experiments (Fig. S1).

Fishing experiments with PG3. An alternative protocol to record the force extension response of polymer molecules is the fishing technique, see Fig. 1a. Thereby, the polymer is adsorbed to the AFM tip. The seeming advantage of this approach is that fewer polymer molecules should come in contact with the substrate, which could lead to a higher probability to detect single molecule events. The functionalized AFM tip is incubated in the polymer solution of a concentration $80 \mathrm{mg} / \mathrm{L}$ for $40 \mathrm{~min}$. The force curves are recorded against EF-silica as substrate, whereby the probability to detect an event is about $1 \%$. The fishing technique can be used in the lifting as well as in the contact and noncontact clamping mode. Figure $8 \mathrm{~b}$ shows the respective results at high ionic strength. The force response obtained by fishing is again substantially softer than the ones obtained by nano-handling, and an important lack of reversibility is observed. These results strongly resemble the ones obtained by the pulling technique (Fig. 8a). Similar results are obtained in the lifting or contact clamping mode. The respective results are summarized in the supplement (Figs. S2 and S3). The lifting mode also 
yields substantially softer response than the one obtained by nano-handling, especially at higher ionic strengths. At lower ionic strength, the different techniques yield results that are in good agreement with nano-handling. Irrespective of the mode used, the fishing experiments strongly resemble the pulling experiments.

A similar fishing technique was used earlier to record force-extension relationships of DPs [44]. At low ionic strength, these experimental results agree with the stretching response of an individual DP obtained by the nano-handling technique. At high ionic strengths, however, the reported response is softer and disagrees with the nano-handling experiments. This disagreement probably results from crowding effects within the adsorbed polymer layer. While an increase of the effective Kuhn length with the ionic strength could be established with the nano-handling technique on the single molecule level as well [12], the decrease of the elastic constant reported by Popa et al. [44] with the ionic strength does not reflect a property of individual polymers. That effect strongly resembles the softening reported in Fig. 8, and therefore it is not related to a variation of single molecule properties with the ionic strength, but rather interaction of the stretched polymer molecule with other adsorbed polymers.

Imaging of PG3 on planar substrates. To provide further indications why the pulling experiments yield different results with AF-mica and EF-silica, AFM images of the adsorbed PG3 molecules on these substrates were recorded. They are shown in Fig. 9. The deposition was carried out with a solution of an ionic strength of $0.1 \mathrm{mM}$ and a polymer concentration was $80 \mathrm{mg} / \mathrm{L}$ during $40 \mathrm{~min}$. These are the same conditions as used for the pulling experiments. The figure compares the image taken in air, and in liquid at an ionic strength of $100 \mathrm{mM}$ and $\mathrm{pH}$ 4.0. For AF-mica, the adsorbed polymers are well separated and they feature rare inter-chain crossings. The pulling experiments carried out with AF-mica have shown good agreement with the nano-handling technique, and therefore they likely reflect the single molecule response. The adsorbed layer on the EF-substrate is much denser, and features numerous inter-chain crossings. In this case, the force response recorded in the pulling experiments did not agree with the nano-handling technique. When the polymer chain is pulled from EF-silica, this chain will be interacting with many other chains. We suspect that interaction between the pulled molecule and other adsorbed chains has the same origin as the observed disagreement between results measured by pulling and nano-handling. The different appearance of the adsorbed layer on the two different substrates is thus likely related to the interaction between the polymer and the substrate. The polymer binds covalently on EF-silica, and for this reason the resulting coverage is much larger. For AF-mica, the surface saturates quickly, probably due to strong electrostatic repulsion between the chains. Moreover, the functionalization is not completely uniform, and that the surface features hot spots, where adsorption is favorable. These hot spots probably correspond to covalent attachment points. A similar situation was found by Cui et al. [34] in 
single molecule peeling experiments featuring force plateaus. These authors also observed single molecule response when the adsorbed layer was dilute and featured few inter-chain crossings, while a denser adsorbed layer did not generate a single molecule response. Surface coverage was also shown to be responsible for a transition between peeling and pulling events [37]. These findings suggest that AFM imaging may help to identify appropriate conditions when single molecule response is to be expected.

Nano-handing and fishing experiments with PG1. To investigate whether the present findings are of wider relevance, we have carried out similar experiments with PG1. The force response of single molecules was measured with the nano-handling technique. These results were compared with profiles obtained by the fishing technique. In both cases, the contact clamping mode was used and the force curves were normalized at $0.25 \mathrm{nN}$. Figure 10 illustrates these results. At an ionic strength of 0.1 $\mathrm{mM}$, the nano-handling and fishing results agree well, indicating that the fishing technique also properly captures the stretching response of a single molecule. At an ionic strength of $100 \mathrm{mM}$, however, one again observes a disagreement between these two techniques. In this case, however, one obtains a stiffer force response with fishing technique. This observation indicates that several molecules might have been picked up at the same time.

Nano-handing and pulling experiments with P2VP. A similar comparison was carried out with $\mathrm{P} 2 \mathrm{VP}$. The polymer was adsorbed from a dilute aqueous solution of $0.5 \mathrm{mg} / \mathrm{L}$ to bare mica or silica. The results are summarized in Fig. 11. The individual polymers were imaged before and after the experiment, and the force profiles were recorded with the nano-handling technique in the lifting mode. These profiles were now normalized at $0.10 \mathrm{nN}$ and compared with the results obtained by the pulling technique. The pulling technique again leads to force profiles, which differ from the ones obtained by nano-handling. While one of these populations shows good agreement with nanohandling, the other population features a softer response. The images shown in Fig. 11a,b,e illustrate the structure of the surface layers in these experiments. While the individual polymers are well separated on the substrate in the nano-handling experiments, they are adsorbed in a dense layer for the pulling experiments. These experiments point towards another difficulty of the nano-handling technique. One must be able to image the polymer in question with the functionalized tip, which is possible for mica, but difficult for silica. One of the reasons that P2VP was chosen for these experiments is that it can still be imaged with relative ease, while other polymers may pose problems in this respect.

Possible Mechanisms. Results obtained by pulling or fishing techniques agree with the ones obtained by the nano-handling technique for some experimental conditions, especially at low ionic strength. Frequently, however, these results disagree, especially at high ionic strengths. Based on the present 
investigations, a single molecule response is obtained by nano-handling, while is far from obvious to obtain such a response from pulling or fishing techniques.

In most cases studied here, pulling and fishing techniques lead to softer response. One possibility to explain these discrepancies is that the molecule is desorbing while it is being pulled. This mechanism would lead to a plateau followed by stretching spike, but such force profiles are hardly observed. However, when the molecule desorbs only little by little, while remaining attached, this mechanism may explain a softer response, since the contour length increases as the polymer is being pulled. This mechanism further rationalizes the influence of the substrate, since the molecules can desorb more easily from a densely adsorbed layer with frequent chain crossings, while desorption will be more difficult from a polymer-free substrate. However, this process will lead to force profiles lacking reversibility. Especially, when a single chain experiment is studied in the non-contact clamping mode, the apparent chain response will become increasingly softer. While this mechanism explains many of the present findings, it does not rationalize the findings reported in Fig. 8.

Another scenario that might lead to different stretching response is the uplift of several adsorbed chains that are entangled with the chain that is being lifted up. In such a situation, one will not only stretch an individual polymer, but elastically deform the entire adsorbed polymer network as well. The force necessary for such a deformation is likely to be substantially smaller than the forces to stretch individual polymer chains, which could lead to the observed softening of the apparent response. This situation might also lead to a reversible force response. For this reason, observing reversibility is not a sufficient criterion to ensure that the stretching of single molecule is being recorded. Moreover, the absence of reversibility may also be induced by conformational transitions within the molecule [53]. This scenario is supported by the importance of the surface coverage, and at low coverage different types of substrates yield the single molecule response.

Picking up and stretching more than one polymer chain may also modify the observed force response. In this case, however, one would expect a stiffer response. Such a response has not been observed in the experiments described here, with the exception of PG1. When several chains are being picked up, this situation can be detected by the lack of collapse on a common master curve. The possibility of stretching several polymer chains has also been pointed out earlier [52,54]. Not surprisingly, the various mechanisms of continuous desorption, entanglement, and picking up several chains can occur simultaneously, and therefore the identification of the detailed mechanism can be non-trivial. 


\section{Conclusion}

The present article compares various techniques to measure the force-extension relationship of individual polymers with the AFM. Reliable force profiles can be obtained by the nano-handling technique, where individual polymer molecules are identified by imaging prior and after the force experiment [12]. However, results obtained by classical pulling or fishing experiments must be interpreted with care. In some situations, such experiments yield the stretching response of individual molecules reliably, especially when the adsorbed polymer layer is dilute. In other situations, however, such experiments may not reflect the expected stretching response, and typically lead to softer force profiles. This softening probably originates from polymer-polymer interactions and entanglements within the adsorbed layer. While the measured force profiles may even pass some the suggested criteria to identify single molecule events, such as being the last event or their collapse upon a master curve $[12,35,49]$, these force profiles may not reflect the force-extension relationship for the stretching process of a single molecule. AFM imaging of the adsorbed layers can be used to address whether such entanglements effects could be important or not, especially in situations when the nanohandling technique cannot be easily implemented.

Supplementary Information. Figures S1, S2, and S3 show the stretching response measured in pulling and fishing experiments with PG3.

Acknowledgement. A useful discussion with Thorsten Hugel is gratefully acknowledged. Support of this research was provided by the National Competence Center in Research (NCCR) for Bio-Inspired Materials, National Research Program "Smart Materials" (NRP 62), other programs of the Swiss National Science Foundation, COST Action CM1101, ETH Zürich, and University of Geneva. 


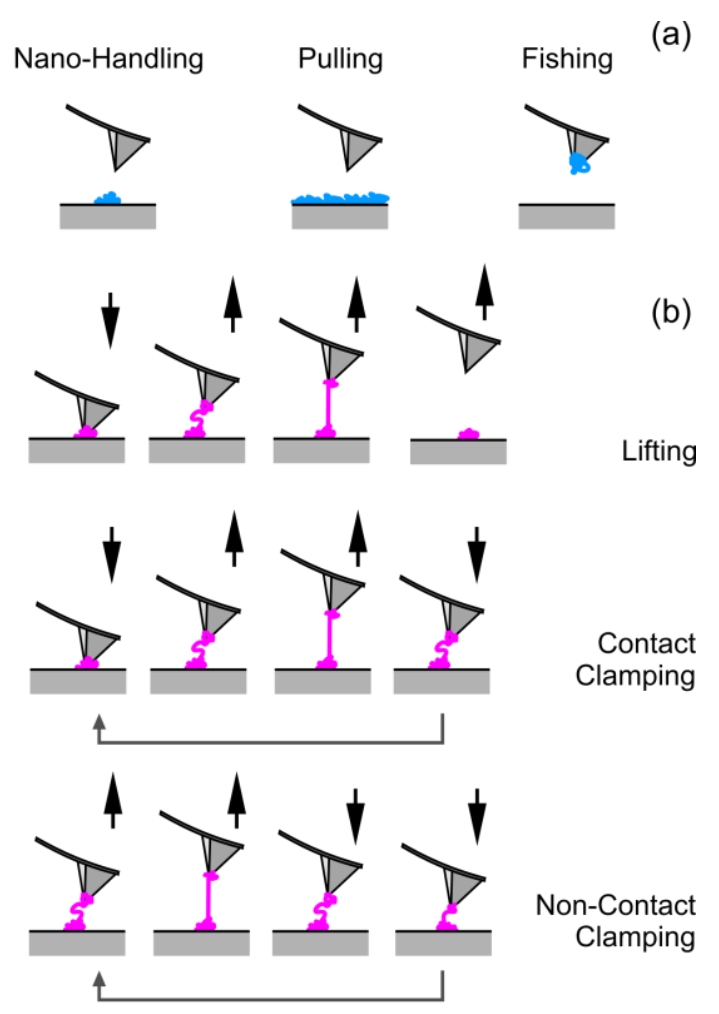

Normalization

(c)

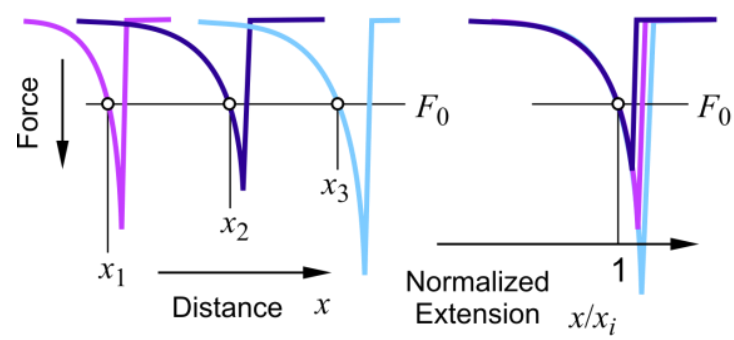

Figure 1. Probing the force response of single polymer molecules with the AFM. (a) Techniques used to pick up single polymer molecules. Nano-handling involves probing a single adsorbed polymer identified by imaging. Pulling lifts single molecules from an adsorbed polymer layer. Fishing uses polymers adsorbed to an AFM tip. (b) Available modes to stretch single polymer molecules. The lifting mode pulls molecules from contact to large distances after rupture. The contact clamping mode repeatedly stretches and relaxes an attached polymer molecule to its maximal extension but contacts the surface in between. The non-contact clamping mode also stretches and relaxes a polymer but avoids contacting the surface in between. (c) Identification of single molecule events by force normalization. Recording stretching events versus distance $x$ and identification of distances $x_{i}$ corresponding to the reference force $F_{0}$ (left) and superposition of the force profiles versus normalized extension $x / x_{i}$. 


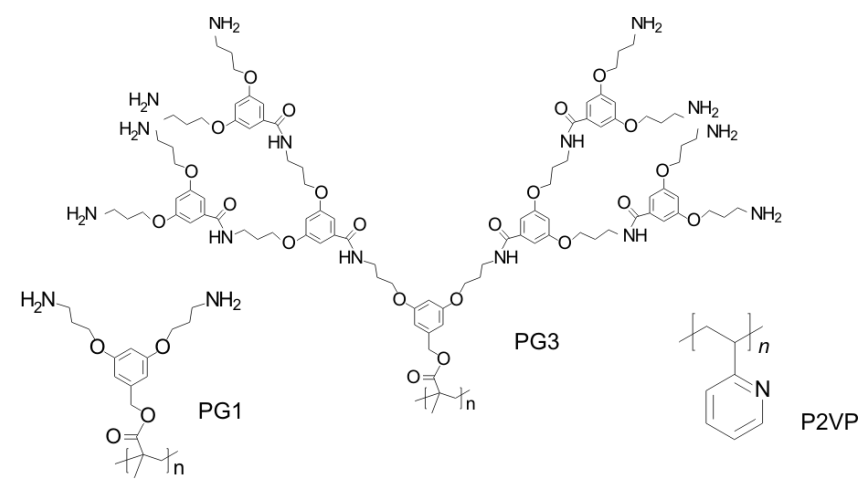

Figure 2. Structural formulas of the polymer used in this study. Amino-functionalized dendronized polymers (DPs) of generation 1 and 3, abbreviated as PG1 and PG3, and poly(2-vinylpyridine) (P2VP). All polymers are positively charged at the conditions investigated. 


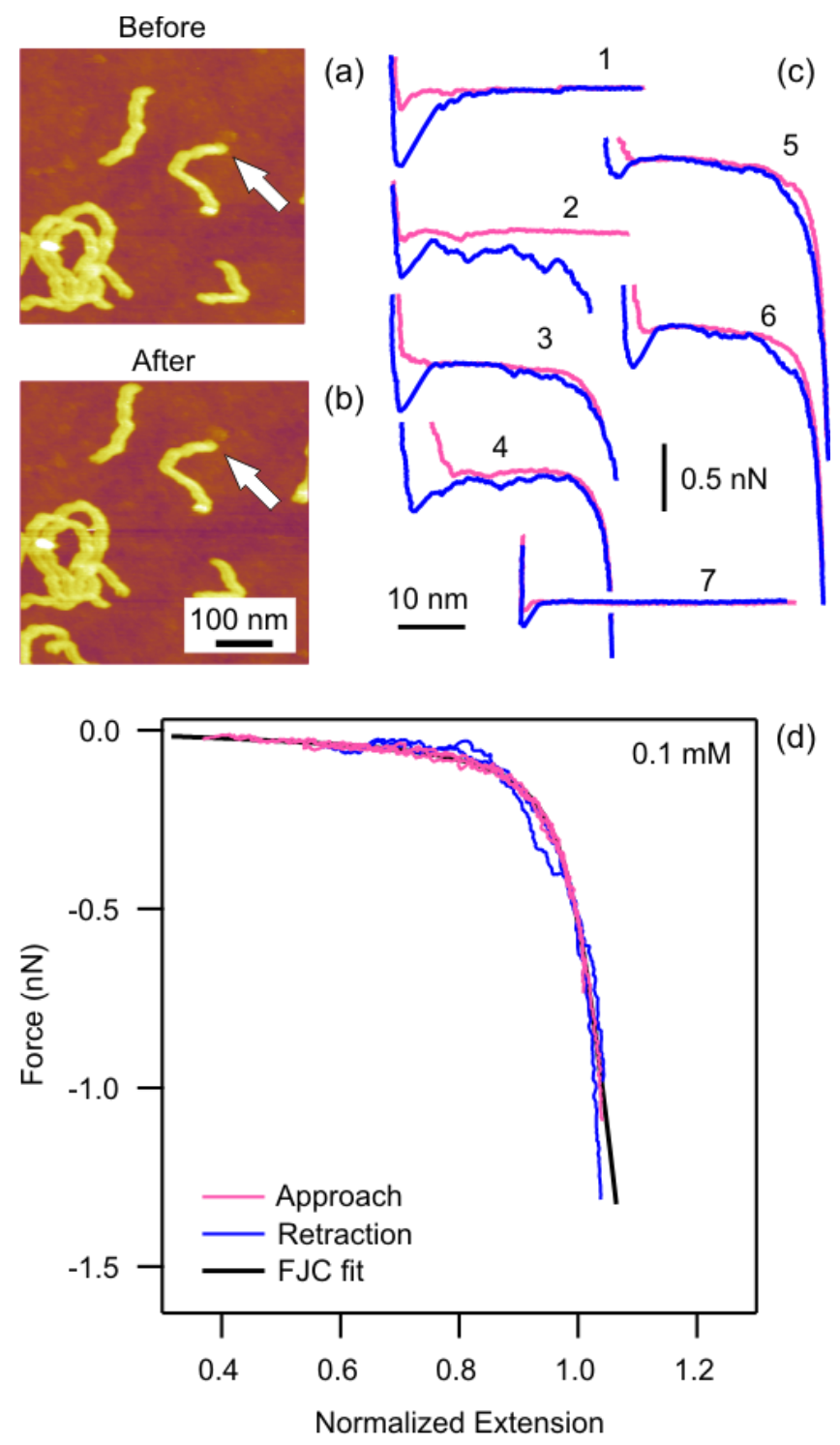

Figure 3. Nano-handling contact clamping experiments for PG3 at pH 4.0 at an ionic strength of 0.1 $\mathrm{mM}$ whereby EF-silica was used as a substrate. Images of the molecules investigated (a) before and (b) after the experiment. The arrow indicates the position where the force profiles were recorded. (c) Typical series of force profiles marked with consecutive numbers. (d) Forces profiles versus normalized extension from approach and retraction parts of the force curves. The solid line is the best fit with the FJC model with the parameters effective Kuhn length $\ell=0.24 \mathrm{~nm}$ and the elasticity constant $K=17 \mathrm{nN}$. 

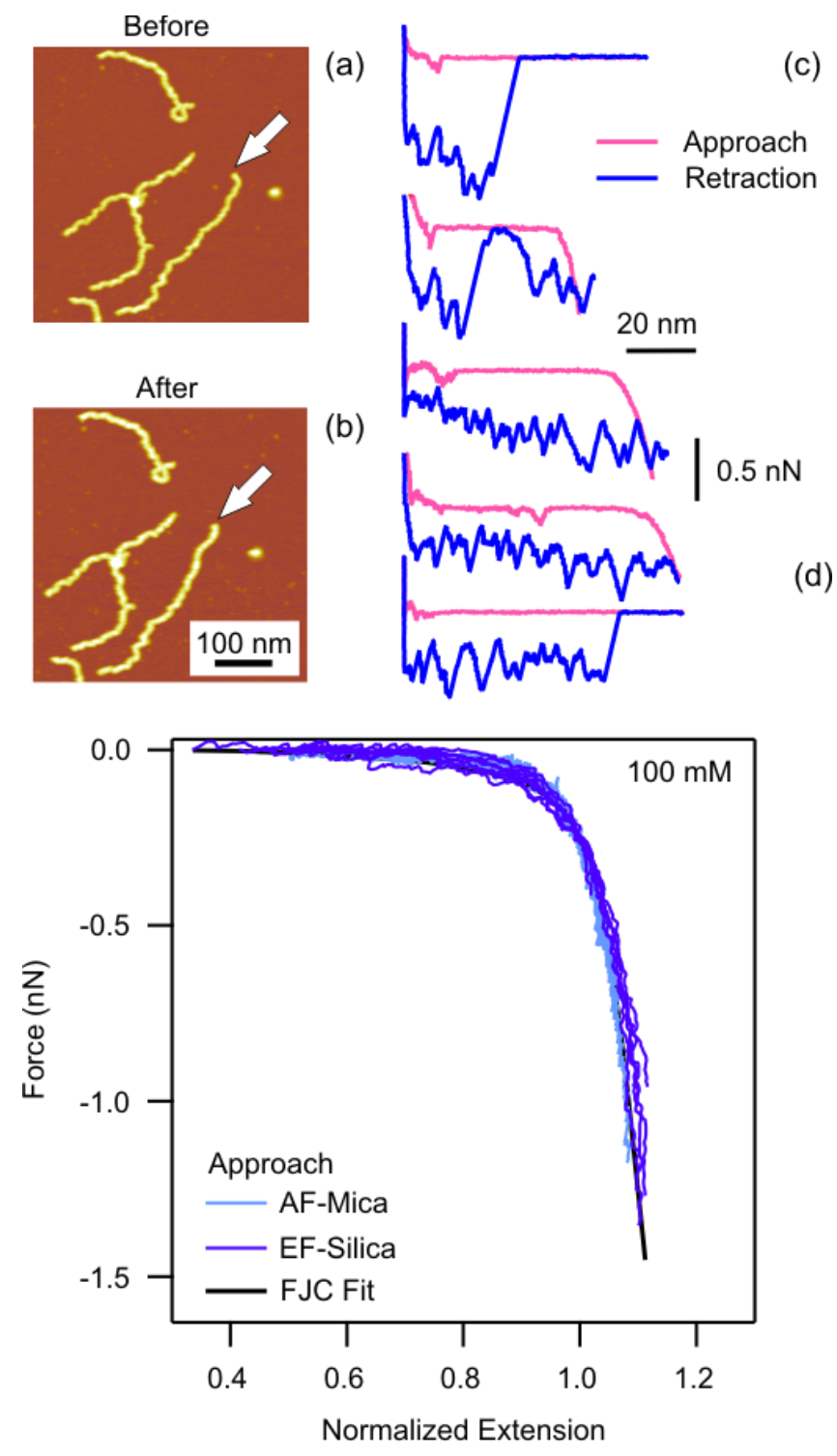

Figure 4. Nano-handling contact clamping experiments for PG3 at pH 4.0 at an ionic strength of $100 \mathrm{mM}$ whereby AF-mica was used as a substrate. Images of the molecules investigated (a) before and (b) after the experiment. The arrow indicates the position where the force profiles were recorded. (c) Typical series of force profiles recorded. (d) Forces profiles versus normalized extension from retraction parts of the force curves for AF-mica and EF-silica substrates. The solid line is the best fit with the FJC model with the parameters effective Kuhn length $\ell=0.27 \mathrm{~nm}$ and the elasticity constant $K=19 \mathrm{nN}$. 

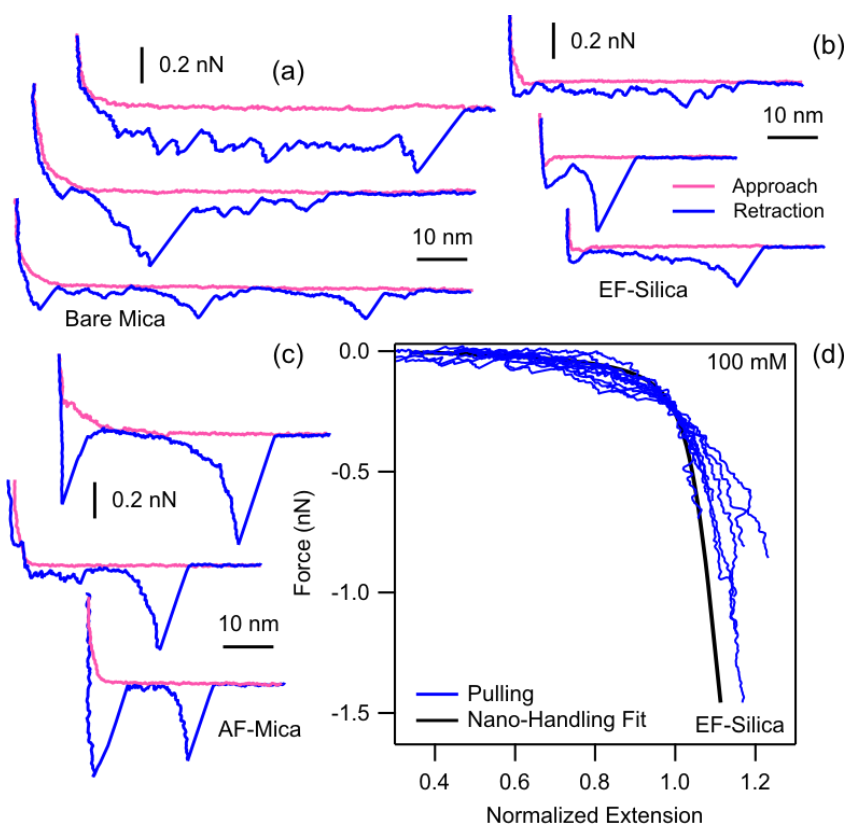

Figure 5. Pulling experiment carried out in the lifting mode for PG3 from different substrates at an ionic strength of $100 \mathrm{mM}$ (a) bare mica, (b) AF-mica, and (c) EF-silica. (d) Force profiles extracted from such events with EF-silica are compared with the best fit of the nano-handling profiles. 

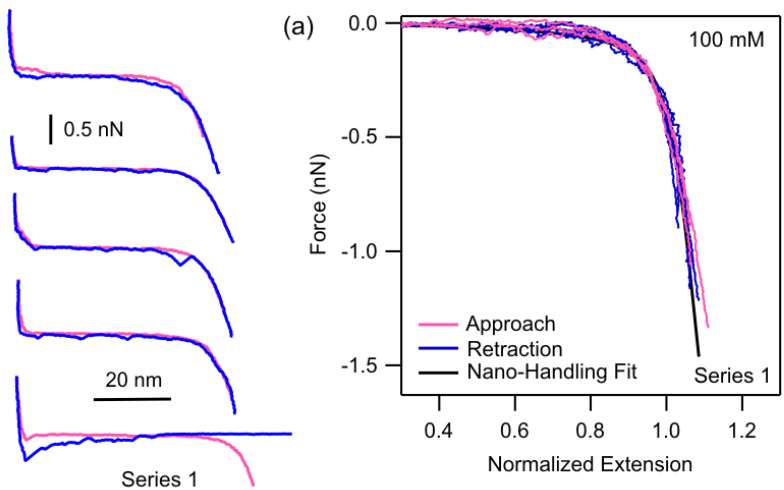

(b)

(c)
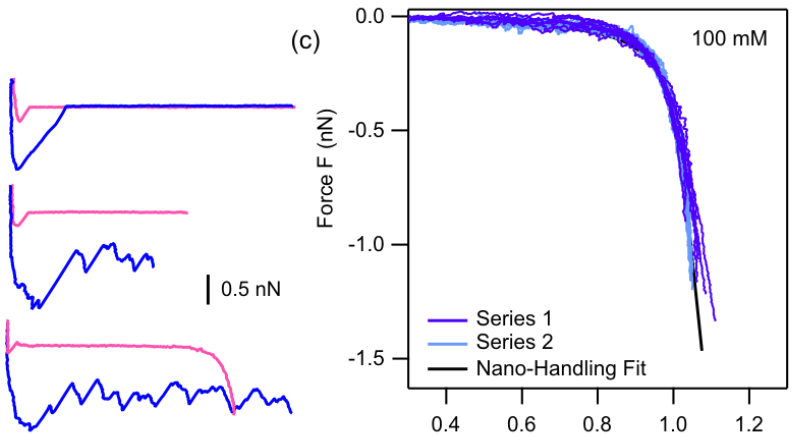

(d)
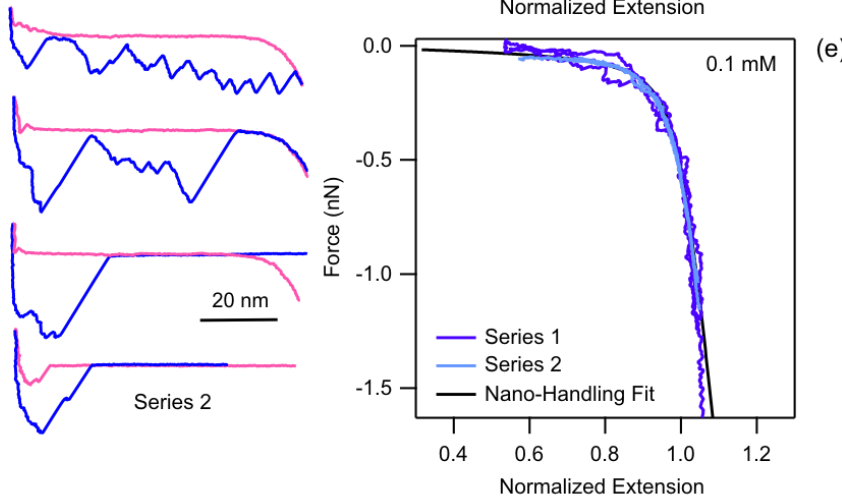

Figure 6. Pulling experiments with contact clamping for PG3 with AF-mica as substrate. (a)

Experimental traces for series 1 at an ionic strength of $100 \mathrm{mM}$, and (b) the corresponding normalized approach and retraction force profiles. Experimental traces for (a) series 1 and (b) series 2 at an ionic strength of $100 \mathrm{mM}$. (c) Comparison of approach and retraction for series 1 and (d) of the two series.

(e) Force profiles for two series recorded at an ionic strength $0.1 \mathrm{mM}$. The force profiles are compared with the best fit of the respective nano-handling profiles. 


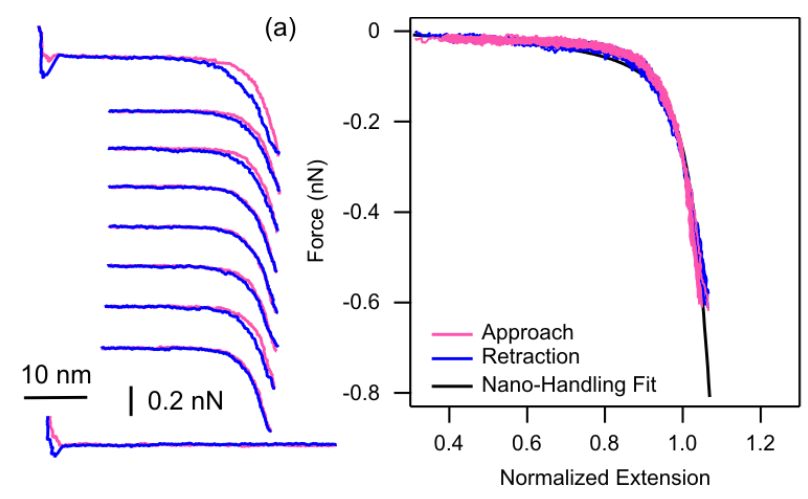

(b)

Figure 7. Pulling experiments with non-contact clamping for PG3 at an ionic strength of $100 \mathrm{mM}$ with AF-mica as substrate. (a) Experimental traces and (b) the resulting force profiles compared with the results obtained from the nano-handling technique. 

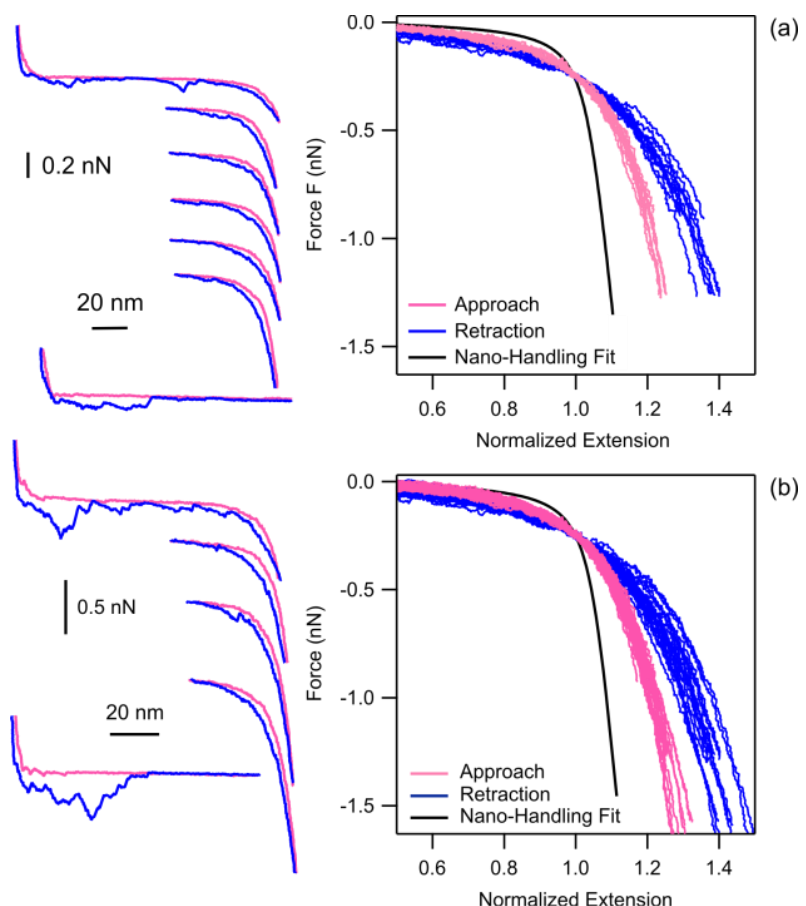

Figure 8. Comparison of pulling and fishing experiments with non-contact clamping for PG3 at an ionic strength of $100 \mathrm{mM}$ with EF-silica as substrate. Experimental traces (left) and the resulting force profiles compared with the results obtained from the nano-handling technique (right). (a) Pulling and (b) fishing experiments. 

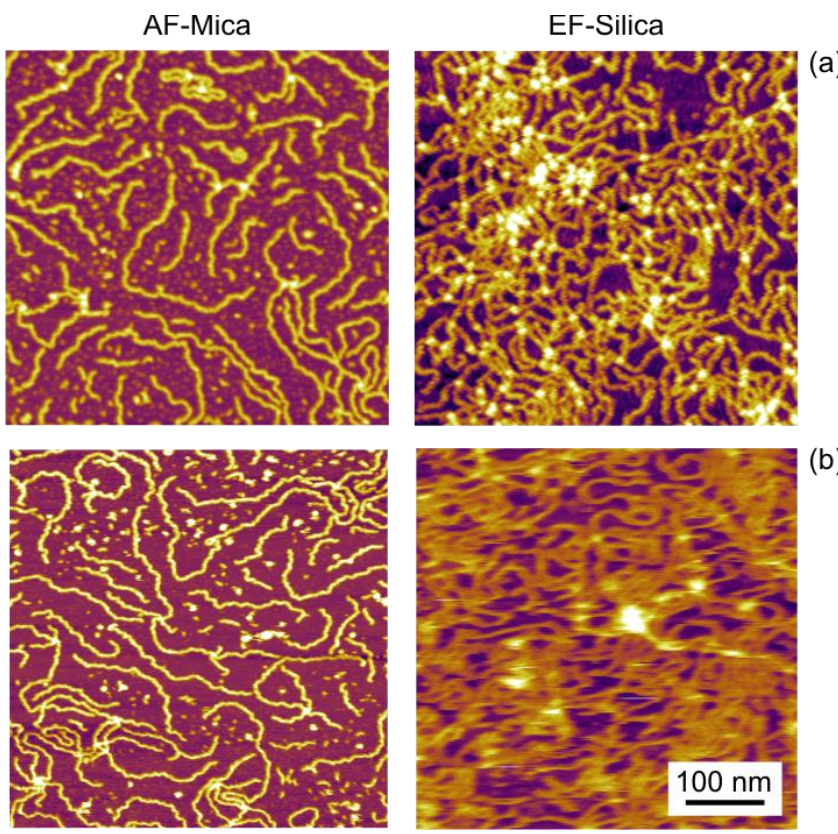

Figure 9. AFM images for adsorbed PG3 on AF-mica and EF-silica. The polymer was adsorbed from a solution of a polymer concentration $80 \mathrm{mg} / \mathrm{L}$ during $40 \mathrm{~min}$. (a) Images taken in air and (b) in solution at an ionic strength of $100 \mathrm{mM}$ and $\mathrm{pH}$ 4.0. 

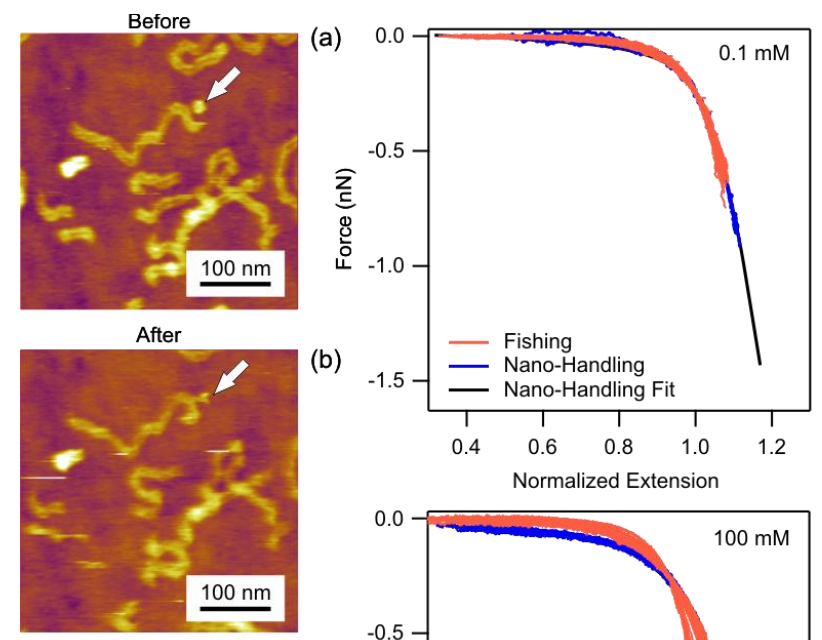

(c)
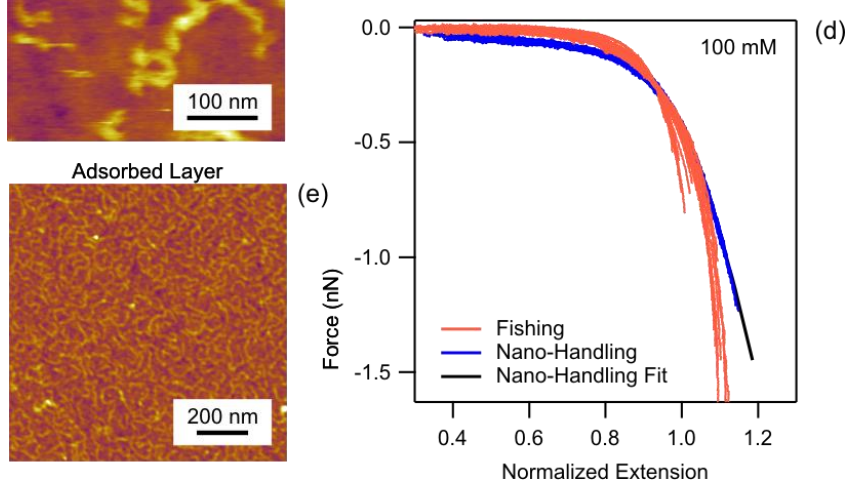

Figure 10. Comparison of nano-handling and fishing experiments in contact clamping mode for PG1 at $\mathrm{pH} 4.0$ with EF-silica. Images of the molecules investigated (a) before and (b) after the experiment at an ionic strength of $0.1 \mathrm{mM}$. The arrow indicates the position where the force profiles were recorded. Normalized forces profiles obtained by nano-handling together with best FJC fit and comparison with the fishing experiments for an ionic strength of (c) $0.1 \mathrm{mM}$, and (d) $100 \mathrm{mM}$. (e) Images of the adsorbed layers prepared with a polymer solution of $80 \mathrm{mg} / \mathrm{L}$. 

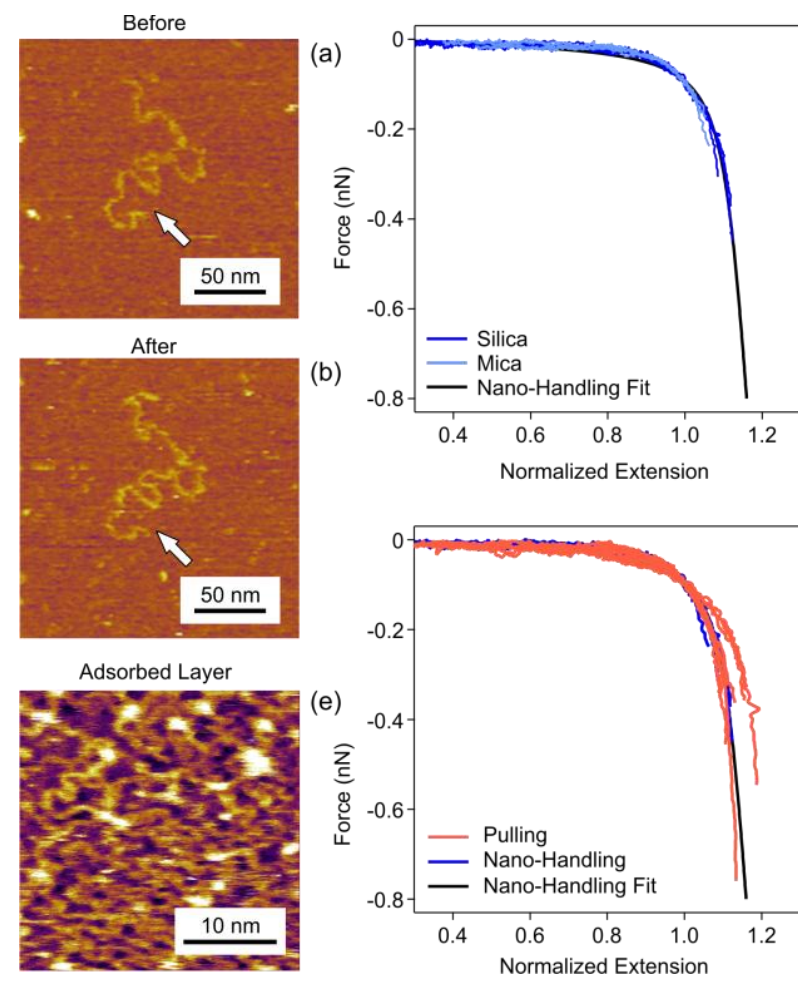

(c)

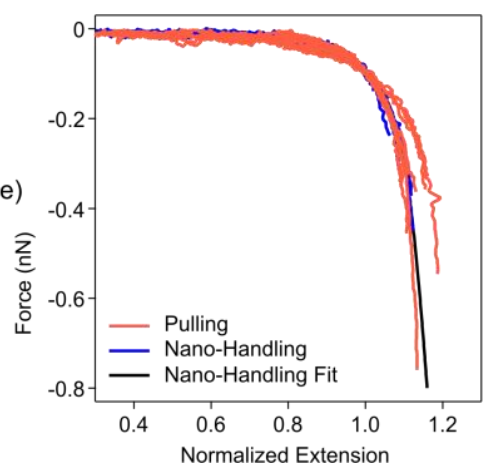

(d)

Figure 11. Comparison of nano-handling and pulling experiments carried out in the lifting mode for $\mathrm{P} 2 \mathrm{VP}$ at an ionic strength of $1.0 \mathrm{mM}$ and $\mathrm{pH}$ 3.0. Images of the molecules adsorbed on mica substrates from a solution with a polymer concentration of $0.5 \mathrm{mg} / \mathrm{L}$ investigated (a) before and (b) after the nano-handling experiment. The arrow indicates the position where the force profiles were recorded. (c) Normalized force profiles obtained by nano-handling are compared for silica and mica and their best FJC fit. (d) Normalized force profiles obtained by nano-handling are compared the best FJC fit and the ones obtained from pulling experiments obtained on silica, mica, and AF-mica. (e) Image of an adsorbed layer prepared with a solution with a polymer concentration of $100 \mathrm{mg} / \mathrm{L}$. 


\section{References}

[1] Roiter Y, Minko S, J. Am. Chem. Soc. 2005; 127: 15688-15689.

[2] Kirwan LJ, Papastavrou G, Borkovec M, Behrens SH, Nano Lett. 2004; 4: 149-152.

[3] Valle F, Favre M, de los Rios P, Rosa A, Dietler G, Phys. Rev. Lett. 2005; 95: 158105.

[4] Popa I, Sinha P, Finessi M, Maroni P, Papastavrou G, Borkovec M, Phys. Rev. Lett. 2010; 104: 228301.

[5] Kirwan LJ, Maroni P, Behrens SH, Papastavrou G, Borkovec M, J. Phys. Chem. B 2008; 112: 14609-14619.

[6] Butt HJ, Cappella B, Kappl M, Surf. Sci. Rep. 2005; 59: 1-152.

[7] Wilke P, Helfricht N, Mark A, Papastavrou G, Faivre D, Borner HG, J. Am. Chem. Soc. 2014; 136: $12667-12674$.

[8] Al-Hellani R, Barner J, Rabe JP, Schlüter AD, Chem. Eur. J. 2006; 12: 6542-6551.

[9] Klukovich HM, Kouznetsova TB, Kean ZS, Lenhardt JM, Craig SL, Nat. Chem. 2013; 5: 110114.

[10] Braithwaite GJC, Howe A, Luckham PF, Langmuir 1996; 12: 4224-4237.

[11] Rief M, Gautel M, Oesterhelt F, Fernandez JM, Gaub HE, Science 1997; 276: 1109-1112.

[12] Grebikova L, Maroni P, Zhang BZ, Schlüter DA, Borkovec M, ACS Nano 2014; 8: 2237-2245.

[13] Severin M, Barner J, Kalachev AA, Rabe JP, Nano Lett. 2004; 4: 577-579.

[14] Zou S, Korczagin I, Hempenius MA, Schonherr H, Vancso GJ, Polymer 2006; 47: 2483-2492.

[15] Kaftan O, Tumbiolo S, Dubreuil F, Auzely-Velty R, Fery A, Papastavrou G, J. Phys. Chem. B 2011; 115: 7726-7735.

[16] Kutnyanszky E, Embrechts A, Hempenius MA, Vancso GJ, Chem. Phys. Lett. 2012; 535: 126130.

[17] Li H, Liu B, Zhang X, Gao C, Shen J, Zou G, Langmuir 1999; 15: 2120-2124.

[18] Lee G, Abdi K, Jiang Y, Michaely P, Bennett V, Marszalek PE, Nature 2006; 440: 246-249.

[19] Shi WQ, Zhang YH, Liu CJ, Wang ZQ, Zhang X, Langmuir 2008; 24: 1318-1323.

[20] Hugel T, Grosholz M, Clausen-Schaumann H, Pfau A, Gaub H, Seitz M, Macromolecules 2001; 34: 1039-1047.

[21] Hugel T, Seitz M, Macromol. Rapid Commun. 2001; 22: 989-1016.

[22] Zhang X, Liu CJ, Wang ZQ, Polymer 2008; 49: 3353-3361.

[23] Radiom M, Honig CDF, Walz JY, Paul MR, Ducker WA, J. Appl. Phys. 2013; 113:

[24] Merkel R, Nassoy P, Leung A, Ritchie K, Evans E, Nature 1999; 397: 50-53.

[25] Kohler M, Karner A, Leitner M, Hytonen VP, Kulomaa M, Hinterdorfer P, Ebner A, Molecules 2014; 19: 12531-12546.

[26] Raman S, Utzig T, Baimpos T, Shrestha BR, Valtiner M, Nat. Commun. 2014; 5: 
[27] Oesterhelt F, Rief M, Gaub HE, New J. Phys. 1999; 1: 6.1-6.11.

[28] Zhang WK, Zou S, Wang C, Zhang X, J. Phys. Chem. B 2000; 104: 10258-10264.

[29] Shi WQ, Wang ZQ, Cui SX, Zhang X, Bo ZS, Macromolecules 2005; 38: 861-866.

[30] Grebikova L, Maroni P, Muresan L, Zhang BZ, Schlüter DA, Borkovec M, Macromolecules 2013; 46: 3603-3610.

[31] Balzer BN, Gallei M, Hauf MV, Stallhofer M, Wiegleb L, Holleitner A, Rehahn M, Hugel T, Angew. Chem. Int. Edit. 2013; 52: 6541-6544.

[32] Radtchenko IL, Papastavrou G, Borkovec M, Biomacromolecules 2005; 6: 3057-3066.

[33] Friedsam C, Seitz M, Gaub HE, J. Phys. Condes. Matter 2004; 16: S2369-S2382.

[34] Cui S, Liu C, Zhang X, Nano Lett. 2003; 3: 245-248.

[35] Seitz M, Friedsam C, Jöstl W, Hugel T, Gaub HE, ChemPhysChem 2003; 4: 986-990.

[36] Sonnenberg L, Luo YF, Schlaad H, Seitz M, Colfen H, Gaub HE, J. Am. Chem. Soc. 2007; 129: $15364-15371$.

[37] Scherer A, Zhou CQ, Michaelis J, Brauchle C, Zumbusch A, Macromolecules 2005; 38: 9821 9825.

[38] Oberhauser AF, Marszalek PE, Erickson HP, Fernandez JM, Nature 1998; 393: 181-185.

[39] Kuhner F, Erdmann M, Sonnenberg L, Serr A, Morfill J, Gaub HE, Langmuir 2006; 22: 1118011186.

[40] Iliafar S, Wagner K, Manohar S, Jagota A, Vezenov D, J. Phys. Chem. C 2012; 116: 1389613903.

[41] Ludwig M, Rief M, Schmidt L, Li H, Oesterhelt F, Gautel M, Gaub HE, Appl. Phys. A Mater. Sci. Process. 1999; 68: 173-176.

[42] Muller DJ, Heymann JB, Oesterhelt F, Moller C, Gaub H, Buldt G, Engel A, Biochim. Biophys. Acta-Bioenerg. 2000; 1460: 27-38.

[43] Sonnenberg L, Parvole J, Borisov O, Billon L, Gaub HE, Seitz M, Macromolecules 2006; 39: 281-288.

[44] Popa I, Zhang B, Maroni P, Schlüter AD, Borkovec M, Angew. Chem. Int. Ed. 2010; 49: 42504253.

[45] Li H, Zhang W, Xu W, Zhang X, Macromolecules 2000; 33: 465-469.

[46] Rief M, Pascual J, Saraste M, Gaub HE, J. Mol. Biol. 1999; 286: 553-561.

[47] Zhang W, Xu Q, Zou S, Li H, Xu W, Zhang X, Shao Z, Kudera M, Gaub HE, Langmuir 2000; 16: 4305-4308.

[48] Marszalek PE, Oberhauser AF, Pang YP, Fernandez JM, Nature 1998; 396: 661-664.

[49] Janshoff A, Neitzert M, Oberdorfer Y, Fuchs H, Angew. Chem. Int. Edit. 2000; 39: 3213-3237.

[50] Guo Y, van Beek J, Zhang B, Colussi M, Walde P, Zhang A, Kröger M, Halperin A, Schlüter AD, J. Am. Chem. Soc. 2009; 131: 11841-11854. 
[51] Zhang B, Wepf R, Fischer K, Schmidt M, Besse S, Lindner P, King BT, Sigel R,

Schurtenberger P, Talmon Y, Ding Y, Kröger M, Halperin A, Schlüter AD, Angew. Chem. Int. Edit. 2011; 50: 737-740.

[52] Papastavrou G, Kirwan LJ, Borkovec M, Langmuir 2006; 22: 10880-10884.

[53] Rief M, Fernandez JM, Gaub HE, Phys. Rev. Lett. 1998; 81: 4764-4767.

[54] Valiaev A, Lim DW, Oas TG, Chilkoti A, Zauscher S, J. Am. Chem. Soc. 2007; 129: 64916497. 